ARTICLE

\title{
Histone H1-mediated epigenetic regulation controls germline stem cell self-renewal by modulating H4K16 acetylation
}

Jin Sun ${ }^{1, \star}$, Hui-Min Wei ${ }^{2, \star}$, Jiang $X^{1,3, \star}$, Jian-Feng Chang ${ }^{2}$, Zhihao Yang ${ }^{1}$, Xingjie Ren ${ }^{1}$, Wen-Wen Lv ${ }^{1}$,

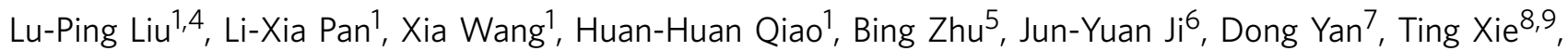
Fang-Lin $\mathrm{Sun}^{2} \&$ Jian-Quan $\mathrm{Ni}^{1}$

Epigenetics plays critical roles in controlling stem cell self-renewal and differentiation. Histone $\mathrm{H} 1$ is one of the most critical chromatin regulators, but its role in adult stem cell regulation remains unclear. Here we report that $\mathrm{H} 1$ is intrinsically required in the regulation of germline stem cells (GSCs) in the Drosophila ovary. The loss of H1 from GSCs causes their premature differentiation through activation of the key GSC differentiation factor bam. Interestingly, the acetylated $\mathrm{H} 4$ lysine 16 (H4K16ac) is selectively augmented in the $\mathrm{H} 1$-depleted GSCs. Furthermore, overexpression of mof reduces $\mathrm{H} 1$ association on chromatin. In contrast, the knocking down of mof significantly rescues the GSC loss phenotype. Taken together, these results suggest that $\mathrm{H} 1$ functions intrinsically to promote GSC self-renewal by antagonizing MOF function. Since $\mathrm{H} 1$ and $\mathrm{H} 4 \mathrm{~K} 16$ acetylation are highly conserved from fly to human, the findings from this study might be applicable to stem cells in other systems.

\footnotetext{
${ }^{1}$ School of Medicine, Tsinghua University, Beijing 100084, China. ${ }^{2}$ Research Center for Translational Medicine at East Hospital, School of Life Sciences and Technology, Tongji University, Shanghai 200092/200120, China. ${ }^{3}$ College of Bioengineering, Hubei University of Technology, Wuhan 430068, China.

${ }^{4}$ Tsinghua Fly Center, Tsinghua University, Beijing 100084, China. ${ }^{5}$ Institute of Biophysics, Chinese Academy of Sciences, Beijing 102206, China.

${ }^{6}$ Department of Molecular and Cellular Medicine, College of Medicine, Texas A\&M Health Science Center, College Station, Texas 77843, USA. ${ }^{7}$ Department of Genetics, Harvard Medical School, Boston, Massachusetts 02115, USA. ${ }^{8}$ Stowers Institute for Medical Research, Kansas City, Missouri 64110, USA

${ }^{9}$ Department of Anatomy and Cell Biology, University of Kansas School of Medicine, Kansas City, Kansas 66160, USA. * These authors contributed equally to this work. Correspondence and requests for materials should be addressed to F.-L.S. (email: sfl@tongji.edu.cn) or to J.-Q.N. (email: nijq@tsinghua.edu.cn).
} 
C hromatin is a complex of DNA and proteins within the eukaryotic cell nucleus. The nucleosome is the basic unit of chromatin and consists of five highly positively charged histones called linker histone $\mathrm{H} 1$ (H1), H2A, H2B, H3 and $\mathrm{H} 4$. The core of the nucleosome octamer contains one tetramer of $\mathrm{H} 3$ and $\mathrm{H} 4$ and two dimers of $\mathrm{H} 2 \mathrm{~A}$ and $\mathrm{H}_{2} \mathrm{~B}^{1}$, which undergo a variety of post-translational modifications. These modifications collectively influence the local chromatin structure and correlate with gene transcription ${ }^{2,3}$, and are tightly associated with stem cell self-renewal, differentiation and proliferation ${ }^{4,5}$. $\mathrm{H} 1$ is also believed to be critical for chromatin remodelling through the condensation of nucleosomes ${ }^{6-9}$. In mammals, $\mathrm{H} 1$ controls chromatin dynamics during early embryogenesis ${ }^{10}$, with just a $50 \%$ reduction in $\mathrm{H} 1$ variants causing embryonic lethality with a broad array of phenotypes ${ }^{11}$. In addition, the depletion of $\mathrm{H} 1$ variants could directly block the differentiation of mouse embryonic stem cells ${ }^{12}$. However, the role of $\mathrm{H} 1$ in the regulation of adult stem cells remains to be determined.

One of the major challenges in elucidating the developmental roles of $\mathrm{H} 1$ in vivo is the high degree of heterogeneity, as multiple $\mathrm{H} 1$ variants with redundant functions exist in most species ${ }^{6,13,14}$. With only one version of $\mathrm{H} 1$ expressed post-embryonically ${ }^{15}$ and well-defined stem cells ${ }^{16}$, the Drosophila ovary is a particularly attractive system to study the functions of $\mathrm{H} 1$ in adult stem cell regulation (Supplementary Fig. 1a). In the Drosophila ovary, two or three germline stem cells (GSCs) are located at the anterior end of the germarium, which is situated at the tip of each ovariole. They physically interact with cap cells anteriorly and escort cells (ECs) laterally. GSCs can be recognized by their proximity to the cap cells and the presence of a spherical organelle known as the spectrosome $^{17}$. The immediate differentiating GSC daughters, cystoblasts, also carry a spectrosome, but are distant from cap cells. Further differentiated germ cell cysts contain a branched fusome, which is an identical organelle to the spectrosome with a different morphology ${ }^{17}$. Therefore, GSCs and their differentiated daughters can be followed and studied.

GSC self-renewal is known to be co-ordinately regulated by both extrinsic signals from niche cells and intrinsic factors 16 . In the adult Drosophila ovary, cap cells and anterior ECs form a self-renewal niche ${ }^{18,19}$. GSCs receive extrinsic signals such as Decapentaplegic (Dpp), the Drosophila homologue of the vertebrate bone morphogenetic proteins for their maintenance ${ }^{20}$. Dpp signalling preserves GSC characteristics and suppresses the transcription of bag-of-marbles (bam), which is both necessary and sufficient for the differentiation of the early GSC lineage ${ }^{21,22}$. Chromatic remodelling factors, ISWI and Domino, have also shown to act intrinsically to promote GSC self-renewal by preventing differentiation, indicating that epigenetic regulation is important intrinsically for controlling GSC self-renewal ${ }^{23,24}$. In addition, Bre-containing protein complex and Enok are also required intrinsically to maintain GSC self-renewal ${ }^{25,26}$. Another potential chromatin regulator Stonewall is also involved in the regulation of GSC fate ${ }^{4}$. Various other intrinsic factors, including Mad, Piwi, Scrawny and Eggless, are also important to maintain GSC self-renewal ${ }^{16}$.

In this study, we have first revealed a critical role of $\mathrm{H} 1$ in maintaining GSCs by preventing bam activation. Interestingly, we find that the H4K16ac level is selectively upregulated in the $\mathrm{H} 1$ knockdown GSCs, and that the association of $\mathrm{H} 1$ on chromatin is antagonized by males absent on the first (MOF), a histone acetyltransferase specific for H4K16. Most interestingly, the knocking down of mof significantly suppresses the GSC loss phenotype induced by the depletion of H1. Taken together, we find that a balance between $\mathrm{H} 1$ and H4K16ac in the chromatin is required for the maintenance of GSCs.

\section{Results}

$\mathrm{H1}$ is intrinsically required for the maintenance of GSCs. To overcome the challenge of genetic analysis of $\mathrm{H} 1$ due to its multiple gene copies in the Drosophila genome, we took advantage of the transgenic RNA interference (RNAi) method by targeting the shared coding sequence of the $\mathrm{H} 1$ genes. We generated a transgenic RNAi line (H1KD line) using a recently developed approach that allowed for knocking-down of $\mathrm{H} 1$ in both spatial and temporal manners ${ }^{27}$. In combination with a nanos-GAL4 (nos-GAL4) line that specifically expresses GAL4 in germ cells (Supplementary Fig. 1c,d), all $H 1 \mathrm{KD}$ females (nos-GAL4 $>$ H1KD) failed to lay any eggs, indicating that $\mathrm{H} 1$ is required for oogenesis. As neither the heterozygous nos-GAL4 flies (nos-GAL4/ + ) nor the nos-GAL4-driven green fluorescent protein (GFP) RNAi flies (nos-GAL4 $>$ GFP-KD) showed any defects in GSC maintenance (Supplementary Fig. 1e-i), we used these two genotypes interchangeably as controls for subsequent experiments. In each control germarium from 3-day-old adults, there were two to three round spectrosome-containing GSCs, recognized by $1 \mathrm{~B} 1$ antibody staining (Fig. 1a,d). In contrast, $92 \%$ of nos-GAL4 $>$ H1KD germaria retained only one or zero stem cell immediately adjacent to the cap cells (Fig. 1b,d). We validated the efficiency of $\mathrm{H} 1$ depletion using quantitative reverse transcription-PCR (qRT-PCR), immunostaining and western blot (Fig. 1e-g, Supplementary Fig. 3a,b). As shown in Fig. 1e, the $\mathrm{H} 1$ transcript level in nos-GAL4 $>\mathrm{H} 1 \mathrm{KD}$ ovaries had reduced to $7.4 \%$ of that in the controls. The qRT-PCR result was further confirmed using another set of primers (Supplementary Fig. 3b), and was consistent with the western blot assay (Supplementary Fig. 3a). By immunostaining with an $\mathrm{H} 1$ specific antibody ${ }^{28}, \mathrm{H} 1$ protein was shown to be efficiently and specifically depleted in the germline of nos-GAL4 $>$ H1KD flies, but not in the somatic cells (Fig. 1f,g). To further validate that the GSC loss phenotype is indeed caused by $\mathrm{H} 1$ depletion, we generated two additional transgenic RNAi lines targeting different sections of the coding regions of $\mathrm{H} 1$ and observed similar GSC loss phenotypes when they are expressed using nos-GAL4 (Supplementary Fig. 2d-h). Furthermore, the expression of $\mathrm{H} 1 \mathrm{cDNA}$ that is not sensitive to RNAi significantly rescued the GSC loss phenotype caused by H1KD (Fig. 1c,d and Supplementary Fig. 2a-c). Taken together, these observations demonstrate that $\mathrm{H} 1$ is required intrinsically for GSC maintenance.

As dBigH1 is also expressed in the Drosophila germline ${ }^{15}$, the question is raised as to whether $\mathrm{dBigH} 1$ is involved in the GSC maintenance phenotype we observed with H1 knockdown. We performed qRT-PCR using nos-GAL4 $>$ H1KD fly ovaries, and did not detect a significant decrease in dBigH1 expression (Supplementary Fig. 3a,b and Supplementary Fig. 3h,i). Furthermore, we constructed two independent dBigH1 transgenic RNAi fly lines, which efficiently knocked down dBigH1 expression when driven by nos-GAL4 (Supplementary Fig. 3c), but did not observe any GSC maintenance phenotypes (Supplementary Fig. 3d-f). In addition, antibody staining showed that both $\mathrm{dBigH} 1$ and $\mathrm{H} 1$ were expressed in the germline but in distinct patterns, as $\mathrm{dBigH} 1$ was mostly expressed at later stages of oogenesis, and in the oocyte (Supplementary Fig. 3h) and nurse cells proximal to the oocyte ${ }^{15}$, whereas $\mathrm{H} 1$ was expressed in earlystage germline cells, but not in the oocyte and at a decreased level in nurse cells neighbouring the oocyte (Supplementary Fig. 3g). These results suggest that H1's roles in GSC maintenance do not involve dBigH1.

To determine further if $\mathrm{H} 1$ is required in adult GSCs for their maintenance, we generated $H 1 \mathrm{KD}$ clones in GSCs using a conditional germline FLP-out system ${ }^{29}$ (Supplementary Fig. 1b). This approach allowed us to deplete $\mathrm{H} 1$ in adult germline cells marked by GFP after clone induction (ACI; Fig. 1i). In the 

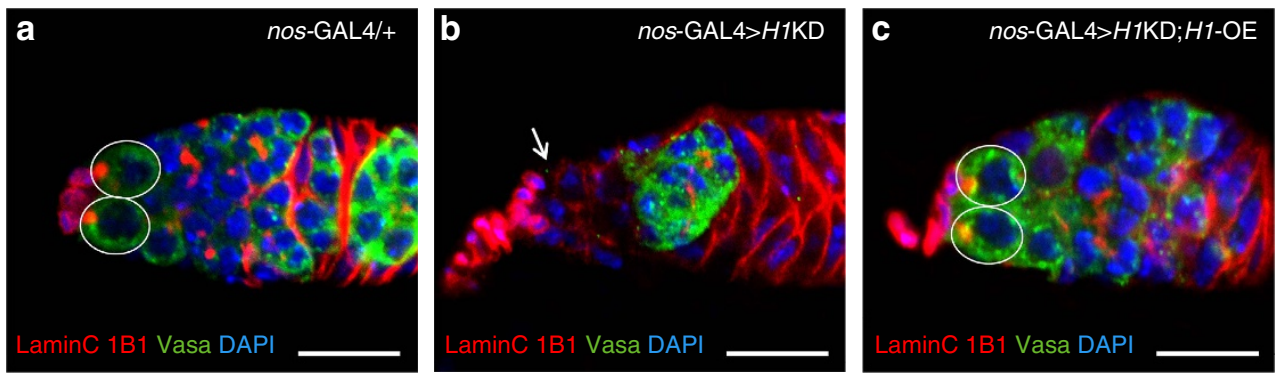

d
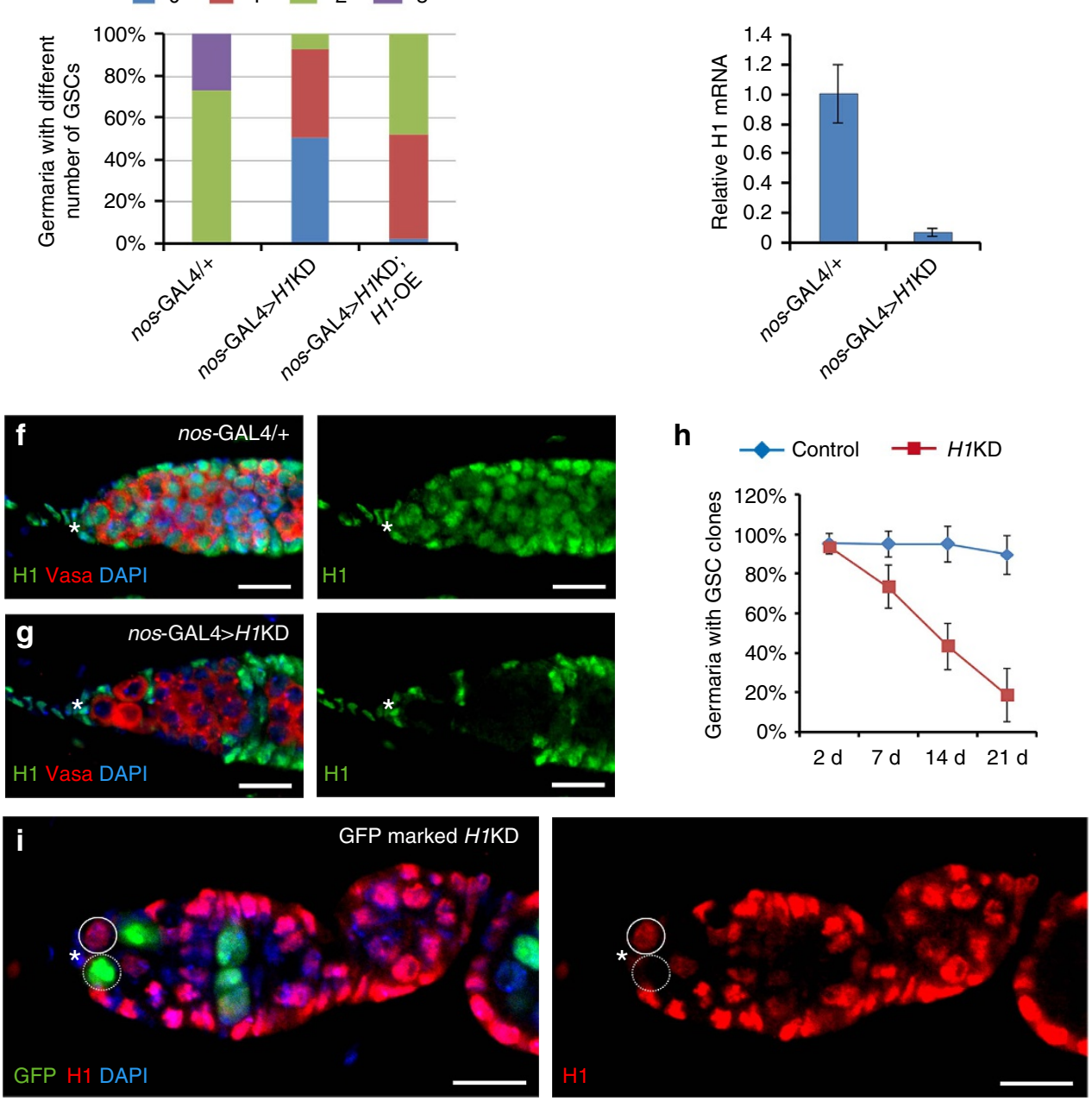

Figure 1 | H1 is required for GSC maintenance intrinsically. (a-c) Germaria are from 3-day-old adult flies, and are stained with 1B1 (red) to reveal spectrosomes/fusomes, anti-Vasa (green) to show the germ cells, 4,6-diamidino-2-phenylindole (DAPI, blue) to show the nuclei and anti-LaminC (also red) to show the nuclear lamina of cap cells and terminal filament cells. Germaria from controls (a) have two GSCs (circles), whereas those from nos-GAL4 > H1KD (b) have no GSC (arrow). (c) H1-OE rescues the loss of GSCs. (d) Column chart showing that nos-GAL4 > H1KD germaria contain fewer GSCs than controls, and that H1-OE can rescue the H1KD GSC loss phenotype. $n=99,97$ and 90 for the three groups, respectively. (e) qRT-PCR results show that the transcription of $\mathrm{H} 1$ in nos-GAL4 $>$ H1KD ovaries is reduced to $7.4 \%$ compared with that in the control. Data are shown as mean \pm s.d. of three independent experiments. (f,g) $\mathrm{H} 1$ (green) is reduced to undetectable level in nos-GAL4 > H1KD germline cells (g) compared with that in the control (f), in 3-day-old adults. (h) The percentage of germaria carrying H1KD GSC clones dramatically decreases with time compared with those carrying wild-type GSC clones. Results are from three experiments and the error bars show the $95 \%$ confidential intervals. (i) H1 is eliminated in GFP-marked H1KD GSCs (broken circles) compared with that in GFP-negative GSCs (circles) 7 days ACI. Scale bars, $10 \mu \mathrm{m}$. Anterior is to the left in this and all subsequent images of germaria. Asterisks indicate the niche cap cells in this and all subsequent images. $d$, day.

controls, the percentage of germaria with GFP-positive GSCs remained at a similar level 3 weeks ACI compared with that at 2 days ACI (Fig. 1h). However, the percentage of germaria with GFP-marked GSCs remarkably declined to $19 \% 3$ weeks ACI (Fig. 1h). These results support the idea that $\mathrm{H} 1$ is intrinsically required for the maintenance of adult GSCs.
Intrinsic depletion of H1 leads to bam activation in GSCs. At the cellular level, the H1 depletion-induced GSC loss phenotype could be caused by apoptosis, premature differentiation, or both. Previous studies showed that $\mathrm{H} 1$ depletion can trigger genomic instability and apoptosis in somatic cells in vivo and in cultured embryonic cells ${ }^{30-33}$. However, we did not observe apoptotic 
GSCs in the H1KD germaria by anti-cleaved caspase- 3 immunostaining (Supplementary Fig. 4a). Furthermore, H1 knockdown cysts have been detected in egg chambers (Fig. 1i and Supplementary Fig. 3i), which is consistent with the apoptosis assay and suggests that $\mathrm{H} 1$ is not required after the germline cells have differentiated. In addition, the GSC loss phenotype was not rescued upon expression of the caspase inhibitor p35 (Supplementary Fig. $4 \mathrm{~b}-\mathrm{d}$ ). These results suggest that the GSC loss phenotype caused by $\mathrm{H} 1$ depletion is unlikely due to apoptosis.

To test whether $\mathrm{H} 1$ depletion causes the premature differentiation of GSCs, we analysed the transcription activation of bam in the H1-depleted GSCs, using bamP-GFP as a reporter ${ }^{22}$. As demonstrated earlier, ectopic expression of bam can trigger GSC premature differentiation, and therefore result in GSC loss ${ }^{34}$. In control germaria, bamP-GFP accumulated primarily in late cystoblasts and differentiating germline cells and was undetectable in the GSCs (Fig. 2a). However, in H1KD germaria, bamP-GFP expression was not only observed in differentiating germline cells but also in GSCs and germline cysts attached to the niche (Fig. 2b,c), suggesting that premature differentiation of GSCs likely contributes to their loss when $\mathrm{H} 1$ is depleted. In addition, qRT-PCR experiments showed that the bam expression level in nos-GAL4 $>$ H1KD ovaries had increased to 1.5 -fold of that in the control ovaries (Fig. 2d). Furthermore, we examined the endogenous protein level of Bam in the H1KD mosaic germarium using immunostaining. In contrast to control GSCs, the intensity of the Bam signal was increased in $H 1 \mathrm{KD}$ GSCs (Fig. 2e,f), further supporting that the ectopic expression of bam after $\mathrm{H} 1$ depletion is associated with GSC loss.

Next, we asked whether the upregulation of bam in $H 1 \mathrm{KD}$ GSCs is caused by compromised Dpp signalling. We examined the expression levels of Dad-lacZ and pMad, two reporters of Dpp signalling activity ${ }^{20,35}$, in the $H 1 \mathrm{KD}$ mosaic germaria. Both Dad-lacZ and pMad were expressed in GFP-positive GSC clones with indistinguishable intensities and patterns 1 week ACI (Supplementary Fig. 4e,f), compared with those in the GFPnegative control GSCs. Therefore, it is likely that $\mathrm{H} 1$ functions downstream of Dpp signalling to repress bam in GSCs.

To test further whether Bam upregulation is the major cause of the stem cell phenotypes in H1KD germaria, we introduced one copy of bam ${ }^{486}$, a mutant allele of bam, into the H1KD GSCs. The $b a m^{\Delta 86}$ heterozygous ovaries show a moderate accumulation of GSC-like cells ${ }^{36}$. As expected, the presence of one copy of $\mathrm{bam}^{486}$ significantly rescued the loss of GSCs in nos-GAL4 $>$ H1KD ovaries, with $40 \%$ of germaria carrying two GSCs compared with $6.5 \%$ of those with two wild-type bam alleles (Fig. $2 \mathrm{~g}-\mathrm{i}$ ). Taken together, these results support the notion that $\mathrm{H} 1$-induced activation of bam expression is largely responsible for the loss of GSCs in $H 1 \mathrm{KD}$ ovaries.

H1KD selectively results in hyperacetylation on H4K16. Loss of $\mathrm{H} 1$ has been shown to alter several histone modifications, including the methylation of $\mathrm{H} 3 \mathrm{~K} 4, \mathrm{H} 3 \mathrm{~K} 9$ and $\mathrm{H} 3 \mathrm{~K} 27$, in specific genes during embryonic stem cell differentiation in vitro ${ }^{12}$. We examined various histone modifications in $H 1 \mathrm{KD}$ germaria driven by nos-GAL4, including the transcriptional repression ones (H3K9me2 and $\mathrm{H} 3 \mathrm{~K} 27 \mathrm{me} 3$ ) and the transcriptional activation ones (H3K4me2, H4K5ac, H4K8ac, H4K16ac and $\mathrm{H} 4 \mathrm{~K} 12 \mathrm{ac}$ ). Interestingly, the level of H4K16ac, a hallmark of hyperactive chromatin, was distinctly upregulated in the germline cells upon depletion of $\mathrm{H} 1$ (Fig. 3a,b), but the other modifications remained unchanged (Supplementary Fig. 5a-1). Using a mosaic analysis in GSCs, we confirmed the upregulation of H4K16ac signals in H1KD GSCs (Fig. 3c,d). The augmented H4K16ac is not due to upregulation of mof, a specific H4K16 acetyltransferase $\mathrm{s}^{37,38}$, as the expression level of mof was not affected upon $\mathrm{H} 1$ depletion (Supplementary Fig. 4g). In addition, the increased H4K16ac was not due to upregulation of H4 level, as the levels of $\mathrm{H} 4 \mathrm{~K} 5 \mathrm{ac}, \mathrm{H} 4 \mathrm{~K} 8 \mathrm{ac}$ and $\mathrm{H} 4 \mathrm{~K} 12 \mathrm{ac}$ were not altered in GSCs compared with those in the control. Therefore, these results suggest that $\mathrm{H} 1$ is required to maintain appropriate levels of $\mathrm{H} 4 \mathrm{~K} 16 \mathrm{ac}$ in GSCs.

MOF antagonizes the association of $\mathrm{H} 1$ on chromatin. As a previous study showed opposite binding trends of $\mathrm{H} 1$ and $\mathrm{H} 4 \mathrm{~K} 16 \mathrm{ac}$ on chromatin ${ }^{39}$, we wondered whether $\mathrm{H} 1$ affects H4K16ac on the chromatin level. The H1 RNAi line that we used could also induce $H 1 \mathrm{KD}$ in somatic tissues (Supplementary Fig. 6a,b), we thus generated $H 1 \mathrm{KD}$ in the salivary gland and found that $H 1 \mathrm{KD}$ leads to the spread of H4K16ac from the $\mathrm{X}$ chromosome to autosomes (Fig. 4a,b). To further test whether $\mathrm{H} 4 \mathrm{~K} 16$ acetylation affects the $\mathrm{H} 1$ level on chromatin in vivo, we increased H4K16ac by overexpressing mof, the H4K16 acetyltransferase. As expected, overexpression of mof resulted in an increase of H4K16ac on chromosomes (Fig. 4c). Importantly, mof overexpression restricted $\mathrm{H} 1$ association on chromatin and triggered chromatin decondensation in salivary gland cells (Fig. 4d). These results show that the overexpression of mof behaves similarly as $H 1 \mathrm{KD}$ to increase $\mathrm{H} 4 \mathrm{~K} 16 \mathrm{ac}$, and prevents $\mathrm{H} 1$ association with the chromatin in vivo. We have also tested an $H 4^{K 16 A}$ mutant gene with the 16th lysine changed to alanine, which makes the residue incapable of being acetylated ${ }^{40}$. Overexpression of this transgene in the salivary gland showed that $\mathrm{H} 4^{\mathrm{K} 16 \mathrm{~A}}$ co-localizes with $\mathrm{H} 1$ on the polytene chromosome, in contrast to the antangonizing pattern of $\mathrm{H} 1$ and $\mathrm{H} 4 \mathrm{~K} 16 \mathrm{ac}$ (Supplementary Fig. 7). These in vivo results suggest the importance of the 16th lysine in the antagonizing relationship between $\mathrm{H} 1$ and $\mathrm{H} 4 \mathrm{~K} 16 \mathrm{ac}$, thus further support a model of mutual repression between MOF and $\mathrm{H} 1$ on H4K16ac.

Antagonism between $\mathrm{H1}$ and MOF in the maintenance of GSC. To determine whether elevated H4K16ac affects GSC maintenance, we used nos-GAL4 to overexpress mof to increase $\mathrm{H} 4 \mathrm{~K} 16 \mathrm{ac}$ in germ cells, including GSCs. As shown in Fig. 5a-c, MOF overexpression indeed caused the GSC loss phenotype, similar to H1KD. Overexpression of MOF in GSCs using the mosaic assay also caused increased H4K16ac expression (Supplementary Fig. 6c) as expected. Similar to the case of MOF overexpression polytene chromosomes, where dissociation of $\mathrm{H} 1$ from chromatin could be observed (Fig. 4e), the level of nuclear $\mathrm{H} 1$ in the GSC was also reduced (Supplementary Fig. 6d). This observation is consistent with a previous study, which showed that knocking down of mof in the mouse embryo leads to an increased level of $\mathrm{H} 1$ (ref. 41). Interestingly, MOF-overexpressing GSC clones also had moderately elevated Bam levels compared with neighbouring control GSCs (Supplementary Fig. 6e), reminiscent of the similar defect in $H 1 \mathrm{KD}$ clones (Fig. 2e). To determine whether the lack of H4K16 acetylation affects GSC differentiation, we also used nos-GAL4 to overexpress $\mathrm{H}_{4}^{\mathrm{K} 16 \mathrm{R}}$, a lysine to arginine mutant that mimics non-acetylated $\mathrm{H} 4 \mathrm{~K} 16$, specifically in the developing germ cells, including GSCs. Strikingly, ectopic expression of $\mathrm{H} 4^{\mathrm{K} 16 \mathrm{R}}$ significantly increased the spectrosome-containing undifferentiated single-germ cells (Fig. 5d). These results suggest that $\mathrm{H} 4 \mathrm{~K} 16 \mathrm{ac}$ is critical for the balance between GSC self-renewal and differentiation.

To test the notion that $\mathrm{H} 1$ and MOF control the H4K16ac levels for balancing self-renewal and differentiation of GSCs, we simultaneously depleted both $\mathrm{H} 1$ and $\mathrm{MOF}$ in germ cells, including GSCs, using nos-GAL4. Double $\mathrm{H} 1$ and MOF 

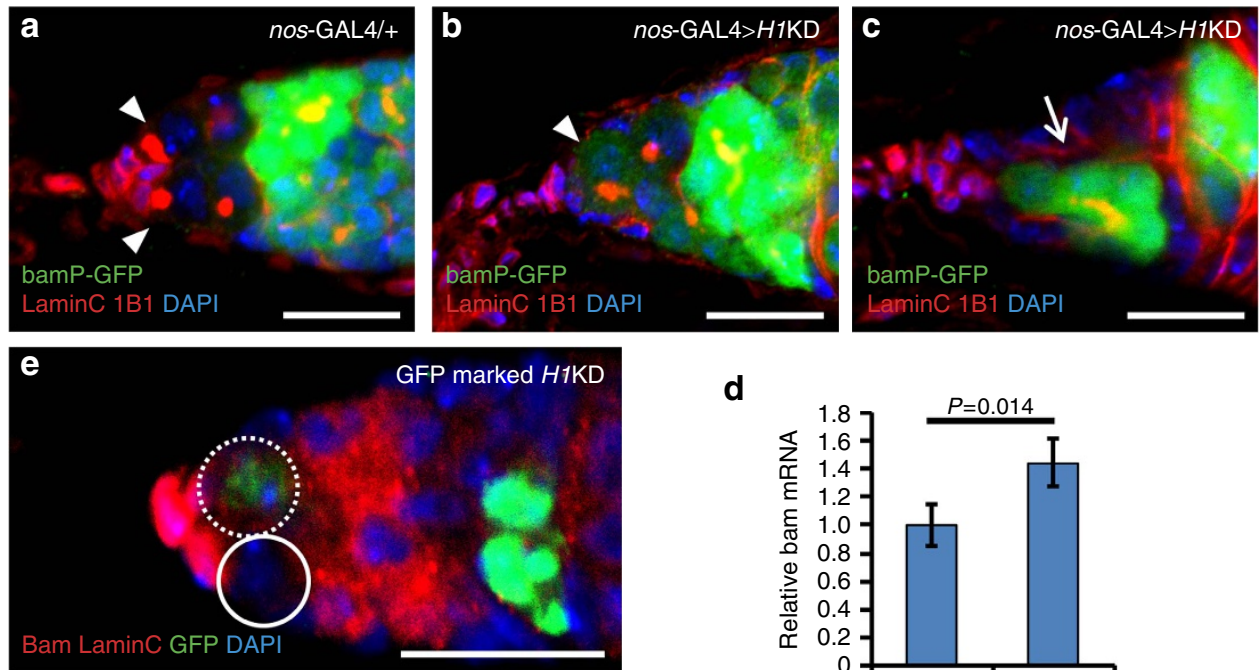

\section{d}
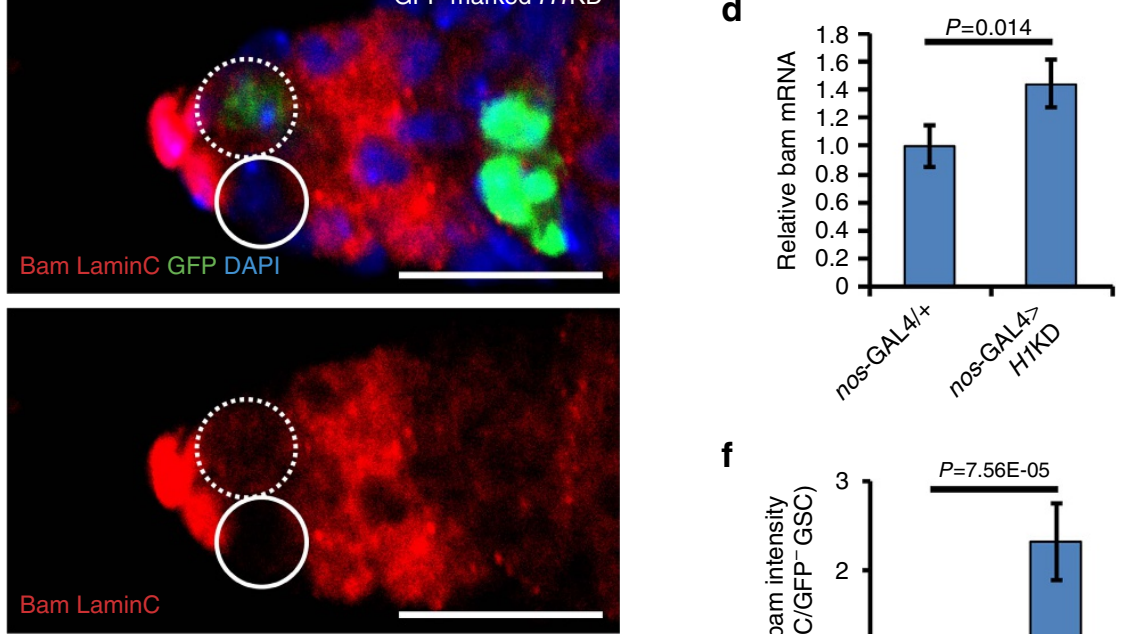

f
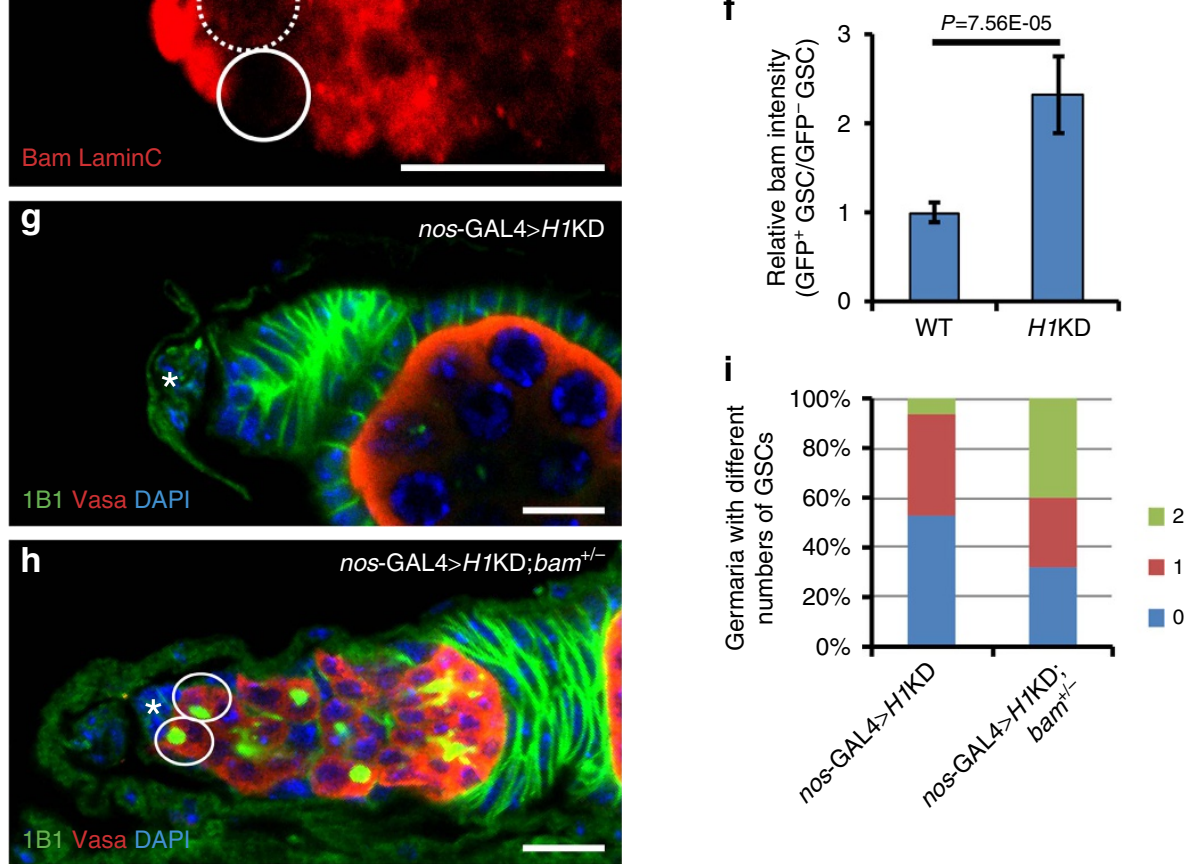

Figure 2 | The loss of H1KD GSCs is due to premature differentiation triggered by bam upregulation. (a-c) bamP-GFP expression (green) is repressed in GSCs of control flies (a), but expands to the GSCs in nos-GAL4>H1KD germaria (arrowhead in $\mathbf{b}$ ). Note that the bamP-GFP-positive germline cells (arrow) neighbouring the cap cells (red nuclear lamina) in c share a branched fusome (red), indicating that premature differentiation of the GSCs occurs in H1KD germaria. Arrowheads in a point to GSCs. (d) qRT-PCR results show that the transcription of bam in nos-GAL4 $>$ H1KD ovaries has increased to 1.5 -fold compared with that in the control $(n=3$, mean \pm s.d.). (e) Bam (red) is increased in the GFP-marked H1KD GSCs (broken circles) compared with that in the GFP-negative control GSCs (circles) 7 days ACI. (f) Quantification of results in e showing that the relative Bam intensity in GFP-marked H1KD GSCs is about twofold higher than that in GFP-negative control GSCs ( $n=5$, mean \pm s.d.). The intensity of Bam in control GSCs is set to 1. Data are evaluated with Student's $t$-test. $(\mathbf{g}, \mathbf{h})$ The bam ${ }^{486}$ heterozygous mutation (h) can suppress GSC loss in nos-GAL4 $>$ H1KD females (g). (i) Quantification of results in $\mathbf{g}$, h showing that the bam ${ }^{486}$ heterozygous mutation can rescue the GSC reduction in H1KD germaria. $n=61$ and $n=25$ for nos-GAL4 $>$ H1KD and nos-GAL4 > H1KD; bam ${ }^{+/-}$groups, respectively. Scale bars, $10 \mu \mathrm{m}$. DAPI, 4,6-diamidino-2-phenylindole.

knockdown (KD) ovaries displayed a significant rescue of the GSC loss phenotype caused by HiKD (Fig. 5e-h and Supplementary Fig. 8a-c). Consequently, females with both H1 and MOF depleted in GSCs laid significantly more eggs than the $\mathrm{H} 1$ and GFP double KD females (Fig. 5i). These results demonstrate the antagonistic relationship between MOF and $\mathrm{H} 1$ in the regulation of GSC self-renewal and differentiation.

\section{Discussion}

In this study, we used Drosophila ovarian GSCs as a model system to reveal that $\mathrm{H} 1$ and MOF antagonize each other's function to control H4K16ac levels, which are critical for GSC self-renewal and differentiation. The loss of H1 in GSCs resulted in their premature differentiation, and the derepression of bam, a critical regulator of GSC differentiation. In addition, our study shows a 

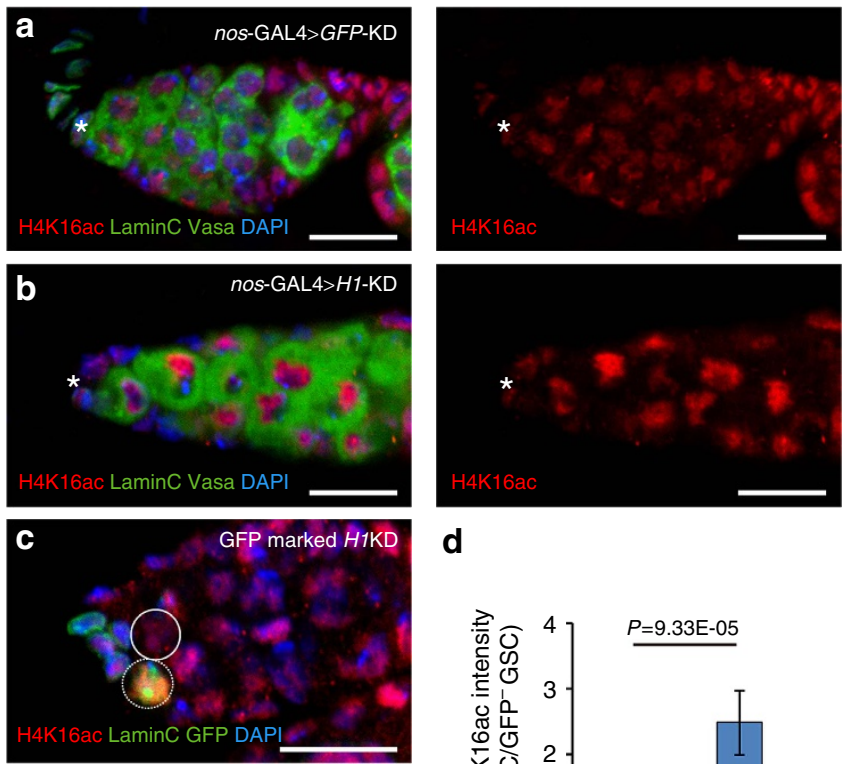

d
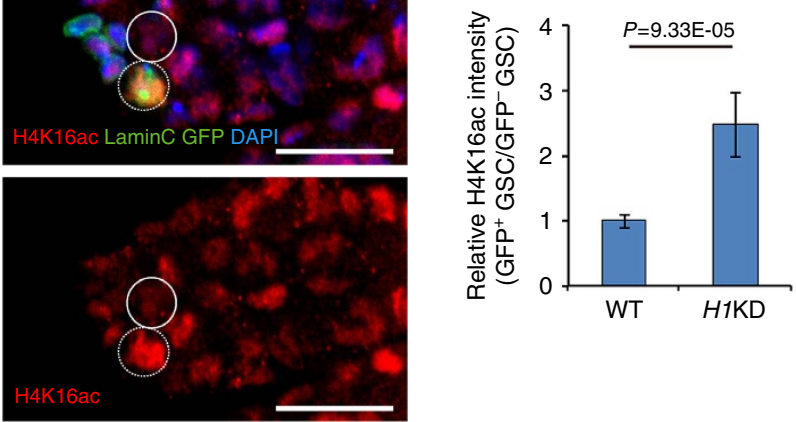

Figure 3 | H1 selectively modulates H4K16ac level. (a,b) H4K16ac levels (red) in the germline are increased in nos-GAL4 > H1KD flies (b) compared with those in controls (a). Anti-Vasa antibody (green; cytoplasm) marks the germline cells, and anti-LaminC (also green; nuclear lamina) marks the cap cells and terminal filaments. (c) H4K16ac level (red) in the GFP-positive (nucleus) H1KD GSC (broken circles) is markedly elevated compared with that in the GFP-negative control GSC (circles). Anti-LaminC (also green; nuclear lamina) marks the cap cells and terminal filaments. (d) Quantification of results in c showing that the relative H4K16ac fluorescent intensity in the GFP-positive H1KD clone is more than twofold higher compared with that in the GFP-negative control GSCs $(n=5$, mean \pm s.d.). The intensity of H4K16ac in control GSCs is set to 1 . Data are evaluated with Student's t-test. Scale bars, $10 \mu \mathrm{m}$. DAPI, 4,6-diamidino-2-phenylindole.

causal relationship between the knockdown of linker $\mathrm{H} 1$ and hyper acetylation on H4K16. Furthermore, this H4K16 hyper acetylation in the germline is specific, as many other core histone modifications do not show obvious changes, which is in contrast to the results of previous studies on somatic tissues ${ }^{30,32}$. The antagonism between $\mathrm{H} 1$ and MOF on chromatin in vivo (Figs 4 and 5), consistent with a previous in vitro study ${ }^{42}$, provides a possible explanation for the transcriptional activation of bam in GSCs.

The acetylation of H4K16 is a critical epigenetic modification in Drosophila as well as in mammals ${ }^{38,43}$. In contrast, other histone modifications, including markers of active and inactive chromatin, showed no obvious changes in ovarian germ cells, although we could not rigorously exclude the possibility that some minor changes may be beyond the detectable level of our assay or limited by the sensitivities of the antibodies. Although it is still not clear whether $\mathrm{H} 4 \mathrm{~K} 16 \mathrm{ac}$ directly affects transcriptional activation in GSCs, our study has established a functional link between $\mathrm{H} 1$, a master transcriptional repressor, and MOF, a critical regulator of active chromatin, in GSCs. This functional interaction between these epigenetic regulators is also strongly supported by experiments showing that double depletion of $\mathrm{H} 1$ and MOF in GSCs can rescue the H1KD phenotype.

Based on our study, we propose a model to explain the functions of $\mathrm{H} 1$ in regulating renewal and differentiation of GSCs in Drosophila (Supplementary Fig. 8d). We propose that $\mathrm{H} 1$ supports GSC maintenance by suppressing regulators of differentiation, such as bam (Fig. 2). This may require at least a certain level of $\mathrm{H} 1$ binding on chromatin, which keeps the genes required for differentiation in an inactive state or at a 'basal level' of transcription, without disrupting stem cell identity. We further propose that transcriptional activator MOF balances the function of $\mathrm{H} 1$ in GSCs by antagonizing the repressive role of $\mathrm{H} 1$. This model is consistent with recent studies showing that MOF resides in a complex with several activators of transcription ${ }^{44}$, and that the loss of MOF in mouse embryonic stem cells resulted in condensed chromatin and differentiation ${ }^{45}$. Interestingly, when $\mathrm{H} 1$ is specifically knocked down in the ECs in the germaria, which form part of the niche for the differentiation of the GSCs and cystoblasts, we have observed an increase of GSC-like, spectrosome-containing cells in the germaria, which resembles an ovarian tumour. Strikingly, this phenotype can also be suppressed by the concomitant knocking down of mof, which also supports our model (Supplementary Fig. 9). Taken together, our results support that linker $\mathrm{H} 1$ is a regulator with a critical function in the regulation of GSC self-renewal, and MOF antagonizes this function through $\mathrm{H} 4 \mathrm{~K} 16$ acetylation. It will be interesting to test whether this mechanism is conserved in mammals, and whether this novel mechanism can explain how dysregulated $\mathrm{H} 1$ contributes ovarian tumorigenesis in humans in the future.

\section{Methods}

Drosophila husbandry and genotype information. The following fly stocks were used in this study: UAS-H1KD RNAi lines (TH00868.N, TH00825.N, and TH00826.N), a UAS-mof RNAi line (TH00870.N), UAS-dBigH1 RNAi lines (TH11322.N and TH11323.N), UAS-GFP RNAi line (TH00782.N), UAS-GFP (TH10512.N), P[nosP-GAL4::vp16] ${ }^{46}, y, h s-F L P$; nos $<S T O P<G A L 4:: V P 16$, UASp-GFP/CyO $\mathrm{O}^{29}, P[\text { bamP-GFP }]^{22}$, Dad-LacZ ${ }^{35}$, bam $^{486}$ (ref. 47), UAS-p35 (ref. 48), 1824-GAL4 (AB1-GAL4; Bloomington \#1824), OK107-GAL4 $\left(\mathrm{w}^{*} ; ; \mathrm{P}\{\mathrm{GawB}\}\right.$ ey ${ }^{\mathrm{OK} 107}$, a gift from Dr Yi Zhong) and c587-Gal4 (Bloomington \#25421).

Sense Oligos of the transgenic RNAi lines are:

TH00782.N: 5'-CCCGAAGGTTATGTACAGGAA-3'

TH00825.N: 5'-AAGCAAGAAGGTAGCCTCTAA-3'

TH00826.N: $5^{\prime}$ - TAGCGAAAGCGTCAAAGGCAA-3

TH00868.N: 5'-ACCAGCGACAGTTGAGAAGAA-3'

TH00870.N: $5^{\prime}$-CTCGACCTCAGCGGTGTCCAA-3'

TH11322.N: 5'-CGGCGAAGTGGTGATGGTTAA-3'

TH11323.N: $5^{\prime}$-ATGGTTAAGCGATCCTTTAAA- $3^{\prime}$.

All Drosophila stocks were maintained at $25^{\circ} \mathrm{C}$ with $60 \%$ humidity on standard cornmeal/sugar/agar media unless otherwise specified.

Transgene constructs and production of transgenic flies. H1, MOF::GFP fusion protein and $\mathrm{H} 4{ }^{\mathrm{K} 16 \mathrm{R}}$ transgene were constructed using a previously described method and a ValiumP vector ${ }^{27}$. Transgenes expressing H1 and MOF::GFP fusion proteins were constructed as follows: the H1-coding sequence was amplified (forward primer: 5' -GGTCTAGAATGGCCATGTCTGATTCTGCAGTTGCA-3'; reverse primer: $5^{\prime}$-AATCTAGATTACTTTTTGGCAGCCGTAGTC- ${ }^{\prime}$ ). The H1 PCR product was digested with $X b a I$ and cloned into a ValiumP vector ${ }^{27}$. The mof-coding sequence was amplified (forward primer: $5^{\prime}$-AACCTAGGATGGCCA TGTCTGAAGCGGAGCTGGA-3'; reverse primer: $5^{\prime}$-AAGAATTCGCCGGAATT TCCCGGAGCT- $3^{\prime}$ ). The PCR product was digested with AvrII/EcoRI and cloned into the ValiumP-GFP vector.

The full cDNA of histone $\mathrm{H} 4$ was amplified (forward primer: $5^{\prime}$-GGTCTAGA ATGGGAATGACTGGTCGTGGTAAAGG-3'; reverse primer: $5^{\prime}$-AAGGATCCT TAACCGCCAAATCCGTAGAG-3'). The PCR product was digested with $X b a \mathrm{I} / E c o$ RI and cloned into the ValiumP-GFP vector. The $H 4^{R 16 R}$ gene was then generated by converting the AAG codon into AGG using the AccuPrime Pfx DNA Polymerase Kit (Invitrogen) according to the manufacturer's instructions. Lysine 16 was replaced with arginine $\left(\mathrm{H} 4^{\mathrm{K} 16 \mathrm{R}}\right)$ in $\mathrm{H} 4-\mathrm{GFP}$.

Transgenic fly lines were produced by injecting the constructs into $y s c v$ nanos-integrase; attP2 or y sc $v$ nanos-integrase; attP40 stocks following the standard procedure ${ }^{27}$. 

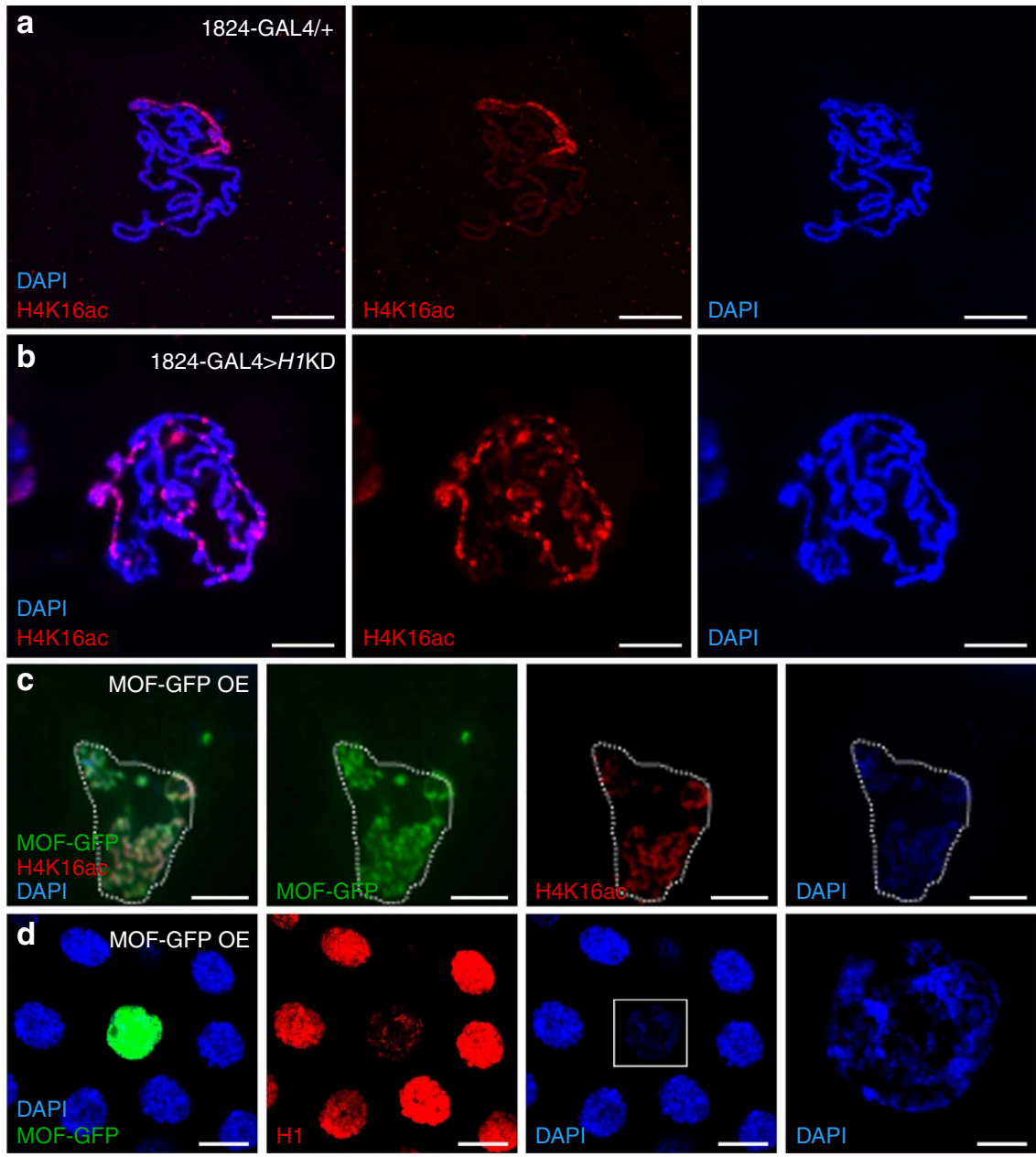

Figure 4 | MOF antagonizes the association of $\mathbf{H 1}$ on chromatin. (a,b) H4K16ac is normally enriched only in the $\mathbf{X}$ chromosome in salivary gland cells of male third-instar larvae (a), but has also accumulated on autosomes in 1824-GAL4-driven H1KD (1824-GAL4>H1KD) polytene chromosomes (b). Note the decondensation of chromatin in $\mathbf{b}$ compared with in a. (c) Overexpression of MOF (green) causes a global increase of H4K16ac (red) on all chromosomes that co-localizes with MOF. Polytene chromosomes are from OK107-GAL4-driven UAS-MOF-GFP flies. (d) In MOF-overexpressing salivary gland cells (green), the association of $\mathrm{H} 1$ on chromatin is greatly attenuated and the decondensation of chromatin is evident, compared with that in the neighbouring control cells. The right panel is a magnified view of the rectangle area in the left panel. Note the similarities between H1KD (b) and MOF-GFP OE (d) phenotypes. Scale bars, $40 \mu \mathrm{m}$ in a and $\mathbf{c}, \mathbf{d} ; 20 \mu \mathrm{m}$ in b. DAPI, 4,6-diamidino-2-phenylindole.

For the overexpression of MOF and $\mathrm{H} 4^{\mathrm{K} 16 \mathrm{~A}}$ in salivary glands, the MOF::GFP- and H4::GFP-coding sequences were subcloned into pUAST vectors, in which MOF::GFP and H4::GFP were flanked by hsp70 basal promoter and the SV40 polyA tail. The $H 4^{K 16 A}$ gene was then generated by converting the 16th codon AAG (Lysin) into GCG (Alanine). The plasmids were then injected into $w^{1118}$ embryos following the standard procedure ${ }^{49}$.

FLP-out and OK107-induced clonal analysis. Clones of RNAi cells in the ovary were generated by FLP/FRT-mediated recombination. To generate GSC clones, $y$, hs-FLP; nos $<S T O P<G A L 4:: V P 16, U A S p-G F P / C y O$ or $y$, hs-FLP; nos $<S T O-$ $P<G A L 4:: V P 16$, UASp-GFP/CyO; Dad-lacZ/TM6B was crossed with the

UAS-H1KD RNAi line (TH00868.N). Adult females of the appropriate genotypes were heat-shocked at $37^{\circ} \mathrm{C}$ for $45 \mathrm{~min} 2$ days after eclosion. The females were then transferred to fresh food at $29^{\circ} \mathrm{C}$, and the ovaries were dissected at days $2,7,14$ and 21 after heat-shock treatment for antibody staining.

Mosaic salivary glands with RNAi cells were generated using the OK107-GAL4 driver. OK107-GAL4 is mostly expressed in the mushroom bodies, but is also expressed in a random manner in a few salivary gland cells ${ }^{40}$. Thus, OK107 was applied to generate random RNAi and overexpression mosaics to study salivary gland cells and polytene chromosomes.

Immunostaining of ovaries. Immunostaining of ovaries followed previously described protocols ${ }^{29}$. Ovaries were dissected in cold PBS, fixed in PBS with $4 \%$ formaldehyde for $15 \mathrm{~min}$ and then washed with PBT (PBS and $0.1 \%$ Triton X-100) five times for $15 \mathrm{~min}$ each. The ovaries were first incubated in $0.5 \%$ goat serum diluted in PBT for $1 \mathrm{~h}$ and then with the appropriate primary antibodies diluted in
PBS at $4{ }^{\circ} \mathrm{C}$ overnight. The samples were then washed with PBT five times for 15 min each, incubated with the appropriate secondary antibodies at $25^{\circ} \mathrm{C}$ for $2 \mathrm{~h}$ then washed with PBT five times for $15 \mathrm{~min}$ each. After the last wash, the stained ovaries were mounted in Fluoromount mounting media (Sigma, F4680). Images were obtained with an inverted Zeiss LSM780 fitted with a ultraviolet laser. The NIS Elements BR programme was used for measurements.

The following primary antibodies were used: mouse monoclonal anti-Hts antibody 1B1 (Developmental Studies Hybridoma Bank (DSHB), 1:100), mouse monoclonal anti-LaminC antibody LC28.26 (DSHB, 1:100), rabbit polyclonal antiVasa (Santa Cruz Biotechnology, sc30210, 1:200), rabbit polyclonal anti-H1 (antiH1C; Fang-Lin Sun $)^{28}$, rabbit polyclonal anti-GFP (Abcam, ab290, 1:1,000), mouse monoclonal anti-GFP (Roche, 1814460,1:50), anti-pMad (Cell Signaling, 9516, 1:50), anti-beta-Galactosidase (Cappel, 55976, 1:1,000), rabbit polyclonal anticleaved Caspase-3 (Cell Signaling, 9661, 1:200), rabbit polyclonal anti-dBigH1 (a gift from Dr Fernando Azorín, 1:40), rabbit polyclonal anti-H4K16ac (Millipore, 07-329, 1:200), rabbit polyclonal anti-H4K12ac (Abcam, ab46983, 1:400), rabbit polyclonal anti-H4K8ac (Cell Signaling, 2594, 1:200), rabbit polyclonal antiH4K5ac (Millipore, 07-327, 1:100), rabbit polyclonal anti-H3K4me2 (Millipore, 07-030, 1:100), rabbit polyclonal anti-H3K27me3 (Cell Signaling, 9733, 1:200), and rabbit polyclonal anti-H3K9me2 (Millipore, 07-212, 1:200). Various secondary antibodies (Jackson ImmunoResearch Laboratories) conjugated with FITC or TRITC were used at 1:200.

To quantify the immunostaining results of the mosaic studies, areas of interes (identified by markers including GFP, LaminC and 4,6-diamidino-2-phenylindole) were selected using the freeform tool in Image and measured to get the Mean Density readings. Then the light intensities of RNAi or overexpression GSC clones were directly compared with that of the neighbouring control GSCs (no GFP 

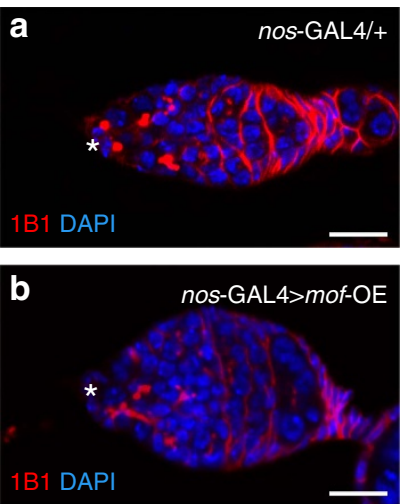

\section{C}
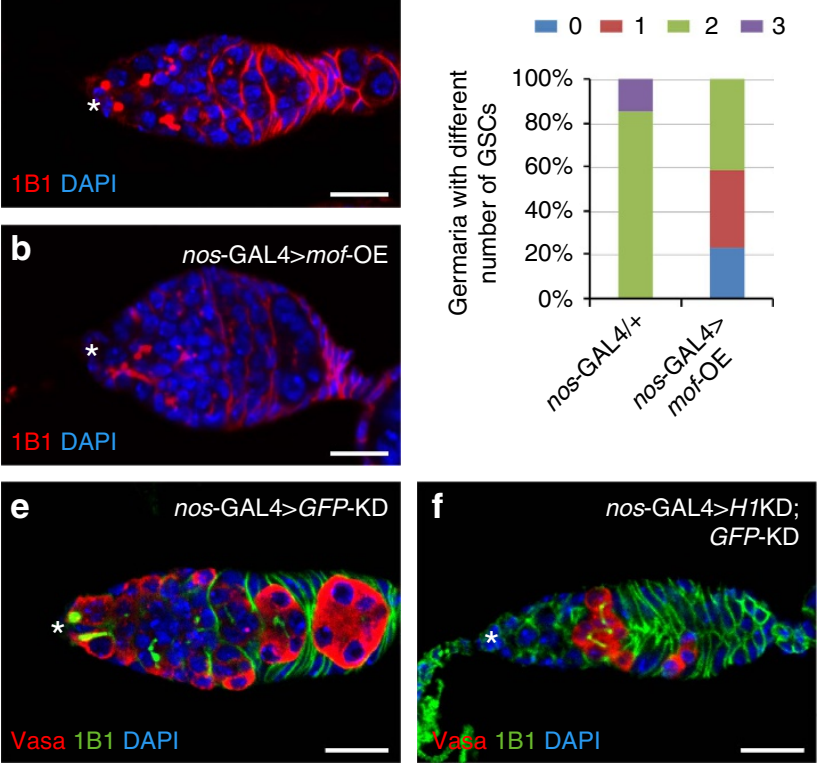

d
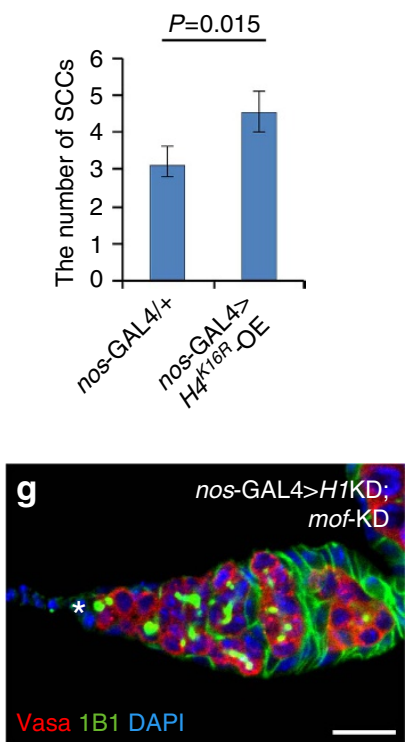

$\mathbf{h}$
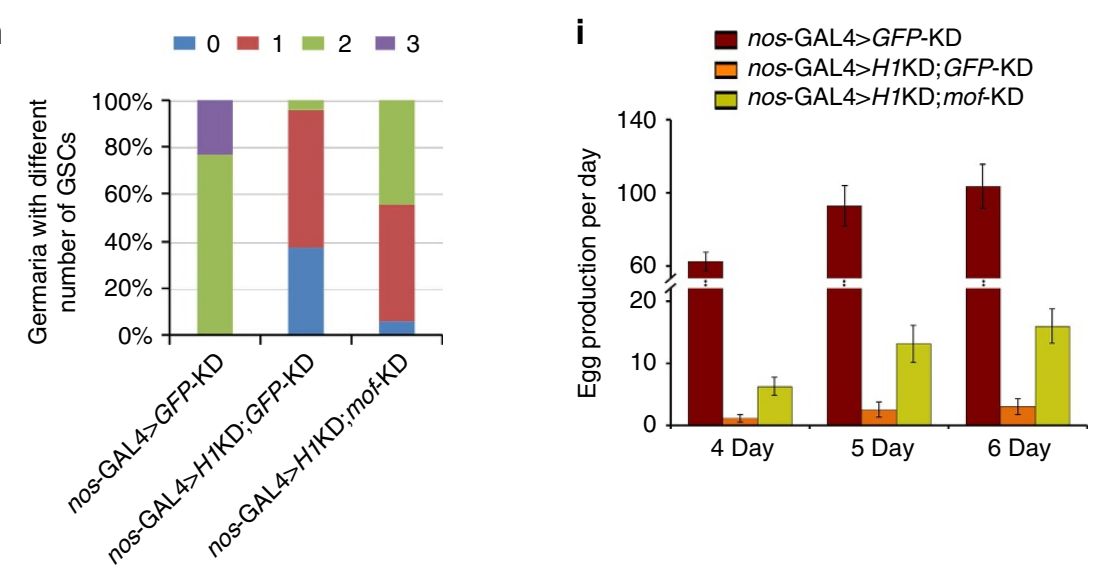

Figure 5 | An antagonism between $\mathbf{H 1}$ and MOF in GSC maintenance. (a,b) Control germarium (a) has two GSCs that can be identified by round spectrosomes (1B1; red) and attachment to the cap cells (asterisk). A loss of GSCs can be detected by $1 \mathrm{~B} 1$ in mof germline overexpression flies (b). Note the prematurely differentiated germline cells neighbouring the cap cells identified by the branched fusome in $\mathbf{b}$. (c) Quantification of the results in $\mathbf{a}, \mathbf{b}$ showing that mof overexpression in the germline causes GSC loss. $n=55$ and $n=70$ for control and experimental groups, respectively. (d) Column chart quantifying the results of the overexpression of a lysine to arginine $\mathrm{H} 4$ mutant ( $\mathrm{H} 4^{\mathrm{K} 16 \mathrm{R}}$ ) on $\mathrm{GSC}$ maintenance. The number of spectrosome-containing undifferentiated single-germ cells (SCCs) has significantly increased in $\mathrm{H} 4{ }^{\mathrm{K} 16 \mathrm{R}}$ germline overexpression germaria, compared with that in nos-GAL4/ + controls. $n=21$ and 24 for the two groups respectively. Data are shown as mean \pm s.e.m. and evaluated with Student's $t$-test. (e-g) H1 and mof double knockdown under the nos driver ( $\mathbf{g}$ ) results in normal numbers of GSCs similar to the control (e), which shows that mof-KD rescues the GSC loss phenotype caused by germline H1KD (f). (h) Quantification of the results in $\mathbf{e}-\mathbf{g}$ showing that mof-KD can rescue the GSC loss phenotype caused by H1KD in the germline. $n=81,114$ and 103 for the three groups, respectively. (i) Quantification of 24-h egg production rates on the 4th, 5th and 6th day after eclosion ( $n=15$ for each group, mean \pm s.d.). Note the $\mathrm{H} 1$ and mof double knockdown significantly increases egg production compared with $\mathrm{H} 1$ and $\mathrm{GFP}$ double knockdown flies. Germaria are from 3-day-old adult flies. Scale bars, $10 \mu \mathrm{m}$. DAPI, 4,6-diamidino-2-phenylindole.

expression). Average light intensity results from five independent pictures were recorded. Error bars denote standard deviation.

Reverse transcription-PCR and qRT-PCR. Total RNA was isolated from Drosophila ovaries 3 day after eclosion using the AxyPrep Multisource Total RNA MiniPrep Kit (Axygen). A total of $1 \mu \mathrm{g}$ RNA was used to create cDNA, using the GoldScript cDNA Kit (Invitrogen) according to the manufacturer's protocol,

followed by PCR to amplify the target sequence. The actin $5 \mathrm{C}$ gene (act5C) served as an internal control.

qRT-PCR was performed using SYBR Premix Ex Taq (TAKARA) and analysed with the iQ5 real-time PCR detection system (Bio-Rad). Results were normalized against rp49 expression.

Primer sequences for reverse transcription-PCR experiments are listed below: act5c-F: 5'-ATACTCCTCCCGACACAAAGC-3'

act5c-R: $5^{\prime}$-CAGGTAGTCGGTCAAATCGC-3'

H1-F: 5'-ggTCTAGAATGGCCATGTCTGATTCTGCAGTTGCA-3'

H1-R: $5^{\prime}$-aactcgagTTACTTTTTGGCAGCCGTAGTC-3'

\author{
mof-F: 5'-CGATTGAGGAGGAGCATGAG-3' \\ mof-R: $5^{\prime}$-CAATTCAACTGGACCTGGTG-3' \\ Primer sequences for qRT-PCR experiments are listed below: \\ rp49-qF: 5'-ATCGGTTACGGATCGAACAAGC-3' \\ rp49-qR: 5'-GTAAACGCGGTTCTGCATGAGC-3' \\ H1-set-1-qF: $5^{\prime}$-CAAAGCTAAGAAGGCTGTGG-3' \\ H1-set-1-qR: 5'-GGCTTCGACTTTATGATTCCAG-3' \\ H1-set-2-qF: $5^{\prime}$-TAAGGGAAAGGGTGCATCTG-3' \\ H1-set-2-qR: 5'-CTTAGAGGCTACCTTCTTGC-3' \\ bam-qF: 5'-CGAGGATACGAACGAAGATGG-3' \\ bam-qR: $5^{\prime}$-GAATTCGAGGAGTGGTGCAG- $3^{\prime}$ \\ dBigH1-qF: 5'-TGAAGGAAAAGAAGGCCTCC-3' \\ dBigH1-qR: 5'-TAGATGCTGGCGGATTATCC-3'.
}

Western blot. Extracts were prepared from 3-day-old adult ovaries. For each genotype, 80 ovaries were collected and lysed in NP-40/300 mM NaCl buffer 
( $1 \% \mathrm{NP}-40,300 \mathrm{mM} \mathrm{NaCl}, 50 \mathrm{mM}$ Tris at $\mathrm{pH} 7.8$, Roche protease inhibitor). The protein concentration of the supernatant was measured by using Bio-Rad Protein Assay Reagent (500-0006). For SDS-polyacrylamide gel electrophoresis, $10 \mu \mathrm{g}$ per lane were loaded. After electrophoresis, proteins were transferred from the gel onto polyvinylidene difluoride membrane (Bio-Rad), then hybridized with primary antibodies at the dilutions indicated: anti-H1C (1:2,000), anti-H3 (Abcam, ab1791, 1:5,000). The secondary antibodies were peroxi-dase-conjugated affinipure goat anti-rabbit IgG $(\mathrm{H}+\mathrm{L})(1: 5,000)$. The ECL detection system (Thermo) was used to detect signals on the blots.

Egg-laying assay. To measure egg-laying rates, virgin females were collected within $6 \mathrm{~h}$ of eclosion, and two (for control vials) or seven (for experimental vials) females were mated to five $w^{1118}$ males. Females were transferred to new vials containing fresh food with several grains of yeast every day for the duration of the experiment. Egg production by individual females was scored by counting the number of eggs laid in successive 24-h periods and dividing by the number of females in each vial. Approximately 15 vials were scored for each group.

Polytene chromosome staining. Immunostaining of third-instar larval polytene chromosomes was performed as previously described ${ }^{50}$. Chromosomes were incubated with rabbit polyclonal anti-H1C (1:500), rabbit polyclonal antiacetylated histone H4K16 (anti-H4K16ac) or mouse monoclonal anti-GFP (MBL M048-3, 1:500) at room temperature for $1 \mathrm{~h}$. After washing with PBS, chromosomes were incubated with a secondary goat anti-mouse antibody conjugated to FITC (1:100) and a goat anti-rabbit antibody conjugated to Texas red (1:100; Jackson ImmunoResearch Laboratories) at room temperature for $2 \mathrm{~h}$. After one PBS wash, the slides were incubated with 4,6-diamidino-2-phenylindole to stain DNA (Sigma, 1:1,000). The immunostained slides were mounted, and the images were taken using a Leica DMI 4000 B inverted microscope (Leica; $\times 40$ model) and processed using Adobe Photoshop software.

\section{References}

1. Luger, K., Mäder, A. W., Richmond, R. K., Sargent, D. F. \& Richmond, T. J. Crystal structure of the nucleosome core particle at 2.8 A resolution. Nature 389, 251-260 (1997).

2. Kouzarides, T. Chromatin modifications and their function. Cell 128, 693-705 (2007).

3. Jenuwein, T. \& Allis, C. D. Translating the histone code. Science 293, 1074-1080 (2001).

4. Maines, J. Z., Park, J. K., Williams, M. \& McKearin, D. M. Stonewalling Drosophila stem cell differentiation by epigenetic controls. Development 134, 1471-1479 (2007).

5. Orkin, S. H. \& Hochedlinger, K. Chromatin connections to pluripotency and cellular reprogramming. Cell 145, 835-850 (2011).

6. Bustin, M., Catez, F. \& Lim, J.-H. The dynamics of histone H1 function in chromatin. Mol. Cell 17, 617-620 (2005).

7. Song, F. et al. Cryo-EM study of the chromatin fiber reveals a double helix twisted by tetranucleosomal units. Science 344, 376-380 (2014).

8. Bednar, J. et al. Nucleosomes, linker DNA, and linker histone form a unique structural motif that directs the higher-order folding and compaction of chromatin. Proc. Natl Acad. Sci. USA 95, 14173-14178 (1998).

9. Misteli, T., Gunjan, A., Hock, R., Bustin, M. \& Brown, D. T. Dynamic binding of histone $\mathrm{H} 1$ to chromatin in living cells. Nature 408, 877-881 (2000).

10. Saeki, H. et al. Linker histone variants control chromatin dynamics during early embryogenesis. Proc. Natl Acad. Sci. USA 102, 5697-5702 (2005).

11. Fan, Y. et al. Histone $\mathrm{H} 1$ depletion in mammals alters global chromatin structure but causes specific changes in gene regulation. Cell 123, 1199-1212 (2005).

12. Zhang, Y. et al. Histone h1 depletion impairs embryonic stem cell differentiation. PLoS Genet. 8, e1002691 (2012).

13. Khochbin, S. Histone H1 diversity: bridging regulatory signals to linker histone function. Gene 271, 1-12 (2001)

14. Talbert, P. B. et al. A unified phylogeny-based nomenclature for histone variants. Epigenetics Chromatin 5, 7 (2012).

15. Pérez-Montero, S., Carbonell, A., Morán, T., Vaquero, A. \& Azorín, F. The embryonic linker histone $\mathrm{H} 1$ variant of Drosophila, dBigH1, regulates zygotic genome activation. Dev. Cell 26, 578-590 (2013).

16. Xie, T. Control of germline stem cell self-renewal and differentiation in the Drosophila ovary: concerted actions of niche signals and intrinsic factors. Wiley Interdiscip. Rev. Dev. Biol. 2, 261-273 (2013).

17. Lin, H., Yue, L. \& Spradling, A. C. The Drosophila fusome, a germline-specific organelle, contains membrane skeletal proteins and functions in cyst formation. Development 120, 947-956 (1994).

18. Wang, X. et al. Histone H3K9 trimethylase Eggless controls germline stem cell maintenance and differentiation. PLoS Genet. 7, e1002426 (2011).

19. Xie, T. \& Spradling, A. C. A niche maintaining germ line stem cells in the Drosophila ovary. Science 290, 328-330 (2000).
20. Xie, T. \& Spradling, A. C. decapentaplegic is essential for the maintenance and division of germline stem cells in the Drosophila ovary. Cell 94, 251-260 (1998).

21. Song, X. et al. Bmp signals from niche cells directly repress transcription of a differentiation-promoting gene, bag of marbles, in germline stem cells in the Drosophila ovary. Development 131, 1353-1364 (2004).

22. Chen, D. \& McKearin, D. Dpp signaling silences bam transcription directly to establish asymmetric divisions of germline stem cells. Curr. Biol. 13, 1786-1791 (2003).

23. Yan, D. et al. A regulatory network of Drosophila germline stem cell selfrenewal. Dev. Cell 28, 459-473 (2014).

24. Xi, R. \& Xie, T. Stem cell self-renewal controlled by chromatin remodeling factors. Science 310, 1487-1489 (2005).

25. Xuan, T. et al. dBrel/dSet1-dependent pathway for histone H3K4 trimethylation has essential roles in controlling germline stem cell maintenance and germ cell differentiation in the Drosophila ovary. Dev. Biol. 379, 167-181 (2013).

26. Xin, T. et al. The Drosophila putative histone acetyltransferase Enok maintains female germline stem cells through regulating Bruno and the niche. Dev. Biol. 384, 1-12 (2013).

27. Ni, J.-Q. et al. A genome-scale shRNA resource for transgenic RNAi in Drosophila. Nat. Methods 8, 405-407 (2011).

28. Ni, J.-Q., Liu, L.-P., Hess, D., Rietdorf, J. \& Sun, F.-L. Drosophila ribosomal proteins are associated with linker histone $\mathrm{H} 1$ and suppress gene transcription Genes Dev. 20, 1959-1973 (2006).

29. Ma, X. et al. Piwi is required in multiple cell types to control germline stem cell lineage development in the Drosophila ovary. PLoS ONE 9, e90267 (2014).

30. Lu, X. et al. Drosophila $\mathrm{H} 1$ regulates the genetic activity of heterochromatin by recruitment of Su(var)3-9. Science 340, 78-81 (2013).

31. Ohsawa, S., Hamada, S., Yoshida, H. \& Miura, M. Caspase-mediated changes in histone $\mathrm{H} 1$ in early apoptosis: prolonged caspase activation in developing olfactory sensory neurons. Cell Death Differ. 15, 1429-1439 (2008).

32. Lu, X. et al. Linker histone H1 is essential for Drosophila development, the establishment of pericentric heterochromatin, and a normal polytene chromosome structure. Genes Dev. 23, 452-465 (2009).

33. Vujatovic, O. et al. Drosophila melanogaster linker histone $\mathrm{dH} 1$ is required for transposon silencing and to preserve genome integrity. Nucleic Acids Res. 40, 5402-5414 (2012).

34. Ohlstein, B. \& McKearin, D. Ectopic expression of the Drosophila Bam protein eliminates oogenic germline stem cells. Development 124, 3651-3662 (1997).

35. Tsuneizumi, K. et al. Daughters against dpp modulates dpp organizing activity in Drosophila wing development. Nature 389, 627-631 (1997).

36. McKearin, D. \& Ohlstein, B. A role for the Drosophila bag-of-marbles protein in the differentiation of cystoblasts from germline stem cells. Development 121, 2937-2947 (1995).

37. Akhtar, A. \& Becker, P. B. Activation of transcription through histone $\mathrm{H} 4$ acetylation by MOF, an acetyltransferase essential for dosage compensation in Drosophila. Mol. Cell 5, 367-375 (2000).

38. Taipale, M. et al. hMOF histone acetyltransferase is required for histone H4 lysine 16 acetylation in mammalian cells. Mol. Cell. Biol. 25, 6798-6810 (2005).

39. Alekseyenko, A. A. et al. Sequence-specific targeting of dosage compensation in Drosophila favors an active chromatin context. PLoS Genet. 8, e1002646 (2012).

40. Lv, W.-W., Wei, H.-M., Wang, D.-L., Ni, J.-Q. \& Sun, F.-L. Depletion of histone deacetylase 3 antagonizes PI3K-mediated overgrowth through the acetylation of histone H4 at lysine 16. J. Cell Sci. 3, 5369-5378 (2012).

41. Lin, C.-J., Conti, M. \& Ramalho-Santos, M. Histone variant H3.3 maintains a decondensed chromatin state essential for mouse preimplantation development. Development 140, 3624-3634 (2013).

42. Shogren-Knaak, M. et al. Histone H4-K16 acetylation controls chromatin structure and protein interactions. Science 311, 844-847 (2006).

43. Gupta, A. et al. The mammalian ortholog of Drosophila MOF that acetylates histone $\mathrm{H} 4$ lysine 16 is essential for embryogenesis and oncogenesis. Mol. Cell. Biol. 28, 397-409 (2008).

44. Wu, L., Li, L., Zhou, B., Qin, Z. \& Dou, Y. H2B ubiquitylation promotes RNA Pol II processivity via PAF1 and pTEFb. Mol. Cell 54, 920-931 (2014).

45. Li, X. et al. The histone acetyltransferase MOF is a key regulator of the embryonic stem cell core transcriptional network. Cell Stem Cell 11, 163-178 (2012).

46. Van Doren, M., Williamson, A. L. \& Lehmann, R. Regulation of zygotic gene expression in Drosophila primordial germ cells. Curr. Biol. 8, 243-246 (1998).

47. Bopp, D., Horabin, J. I., Lersch, R. A., Cline, T. W. \& Schedl, P. Expression of the Sex-lethal gene is controlled at multiple levels during Drosophila oogenesis. Development 118, 797-812 (1993).

48. Hay, B. A., Wolff, T. \& Rubin, G. M. Expression of baculovirus P35 prevents cell death in Drosophila. Development 120, 2121-2129 (1994).

49. Rubin, G. \& Spradling, A. Genetic transformation of Drosophila with transposable element vectors. Science 218, 348-353 (1982). 
50. Liu, L.-P., Ni, J.-Q., Shi, Y.-D., Oakeley, E. J. \& Sun, F.-L. Sex-specific role of Drosophila melanogaster HP1 in regulating chromatin structure and gene transcription. Nat. Genet. 37, 1361-1366 (2005).

\section{Acknowledgements}

We thank Dr Dahua Chen, Dr Thomas Kornberg, Dr Ruth Lehmann, Dr Dennis McKearin, Dr Norbert Perrimon, Dr Tetsuya Tabata, Dr Mark Van Doren, Dr Yi Zhong and the Bloomington Drosophila Stock Center for fly strains and reagents. We thank Dr Fernando Azorín for the anti-dBigH1 antibody. We particularly thank Dr Jerry Workman (Stowers Institute, Kansas City, Missouri, USA) and Dr Babak Javid (School of Medicine, Tsinghua University, Beijing, China) for their kind suggestions on this work. The work was supported by the National Natural Science Foundation of China (31371496), the Ministry of Science and Technology of the People's Republic of China (2015BAI09B03, 2011CB965300 and 2013CB35102), the National Natural Science Foundation of China (31571320, 31301008, 31330043 and 91419304), the TsinghuaPeking Center for Life Sciences and Wuhan Youth Chenguang Program of Science and Technology (2014072704011259).

\section{Author contributions}

J.-Q.N. and F.-L.S. designed the research; J.S., H.-M.W. and J.X. performed the research with contributions from J.-F.C., Z.Y., X.R., W.-W.L., L.-P.L., L.-X.P., X.W., H.-H.Q., B.Z.,
J.-Y.J. and D.Y.; J.S., H.-M.W, J.X., J.-Y.J., D.Y., T.X., F.-L.S. and J.-Q.N. analysed the data; J.-Q.N. wrote the paper with input from F.-L.S., J.-Y.J. and T.X.

\section{Additional information}

Supplementary Information accompanies this paper at http://www.nature.com/ naturecommunications

Competing financial interests: The authors declare no competing financial interests.

Reprints and permission information is available online at http://npg.nature.com/ reprintsandpermissions/

How to cite this article: Sun, J. et al. Histone H1-mediated epigenetic regulation controls germline stem cell self-renewal by modulatingH4K16 acetylation. Nat. Commun. 6:8856 doi: $10.1038 /$ ncomms9856 (2015).

(c) (i) This work is licensed under a Creative Commons Attribution 4.0 International License. The images or other third party material in this article are included in the article's Creative Commons license, unless indicated otherwise in the credit line; if the material is not included under the Creative Commons license, users will need to obtain permission from the license holder to reproduce the material. To view a copy of this license, visit http://creativecommons.org/licenses/by/4.0/ 